\title{
Quelques aspects du cycle continental de l'eau
}

\author{
Joël Noihlan, \\ Météo-France, CNRM, Toulouse
}

\section{INTRODUCTION}

Le cycle continental de l'eau s'effectue dans l'atmosphère et dans le sol. De nombreux processus chimiques sont mis en jeu, dont on trouvera une synthèse dans [28]. La partie atmosphérique correspond au transport de l'eau, en grande partie sous forme de vapeur avec un temps de séjour dans l'atmosphère de l'ordre de la semaine [1]. Les mouvements atmosphériques génèrent des processus de refroidissement sous l'effet par exemple du soulèvement de la masse d'air, entraînant le passage de l'eau de la phase vapeur à la phase liquide. L'eau liquide atmosphérique est concentrée dans les nuages ou sous forme de précipitations neigeuse ou liquide. L'interception du rayonnement solaire par les nuages va entraîner une modification du bilan radiatif des surfaces terrestres et le régime et l'intensité des précipitations va conditionner le bilan hydrique du sol. Lorsque l'eau liquide atteint la surface continentale (sous forme de précipitations ou de rosée), elle est soumise à des mécanismes physiques complexes dans le système constitué par la surface du sol et la végétation : interception par les plantes, ruissellement à la surface du sol, infiltration dans le sol. La branche hydrologique du cycle de l'eau dans le sol se déroule essentiellement en phase liquide et seule une petite partie de l'eau du sol est en mouvement par rapport à l'important stockage de l'eau dans les nappes. Contrairement au transport atmosphérique, les mouvements de l'eau dans le sol sont relativement lents et les temps de séjour peuvent être de plusieurs mois. La capacité de stockage du sol est déterminée par la texture et par la structure du sol. Seule une partie de l'eau est accessible par les plantes, ce qui conduit à la notion de réserve utile du sol utilisée dans la modélisation du bilan hydrique [2]. L'eau est soumise à des mécanismes de rétention au niveau des particules du sol, de percolation sous l'effet de la gravité et de succion par le système racinaire conduisant à la transpiration au niveau des feuilles. L'eau de drainage est transférée au système hydrologique souterrain.

Cet article évoque les divers aspects de la modélisation du cycle continental de l'eau à l'échelle régionale. La modélisation des échanges à l'interface est au cœur de notre propos car elle est le siège des transferts entre les branches atmosphérique et hydrologique du cycle de l'eau. Le paragraphe 2 aborde les différentes manières de modéliser les échanges de masse et d'énergie à l'échelle locale et discute quelques aspects de validation des schémas de surface à partir de données expérimentales. Le paragraphe 3 concerne la modélisation de l'eau à l'échelle régionale en considérant quelques exemples de modélisation atmosphérique à moyenne échelle et en évoquant un projet de modélisation couplée atmosphèrehydrologie appliquée à un grand bassin versant.

\section{II — LA MODÉLISATION DE L'INTERFACE SOL-PLANTE-ATMOSPHERE}

\begin{abstract}
2.1 Les schémas de l'interface sol-plante-atmosphère
L'interface sol/plante-atmosphère assure les échanges de chaleur et d'eau entre les écoulements atmosphériques et hydrologiques. On exprime la conservation de ces échanges à partir du bilan d'énergie et du bilan hydrique. Le bilan d'énergie exprime la transformation du rayonnement net à la surface en chaleur latente (équivalent énergétique de l'évapotranspiration), chaleur sensible (flux par convection entre l'atmosphère et le sol) et conduction dans le sol. Le bilan hydrique exprime la conservation de l'eau dans le sol à partir des échanges d'eau à l'interface (précipitation, ruissellement, évapotranspiration) et la base du réservoir sol (drainage). Bilans énergétique et hydrique sont étroitement couplés à travers l'évapotranspiration.
\end{abstract}

Les flux de surface sont reliés aux propriétés du sol et de la végétation et au contenu en eau du sol. Les bilans radiatifs sont en partie contrôlés par l'albédo du sol et de la végétation. Les échanges de quantité de mouvement et de chaleur dépendent fortement des longueurs de rugosité de la surface (longueurs de rugosité dynamique et thermique). Enfin, la partition de l'énergie radiative diminuée des transferts par conduction dans le sol est contrôlée par le contenu en eau du sol et par la végétation. Ce dernier aspect constitue le point le plus délicat dans les diverses modélisations du continuum sol-plante-atmosphère. Les mécanismes physiques à modéliser font appel à des disciplines très différentes, expliquant la grande diversité des schémas de surface à vocation opérationnelle. On peut néanmoins tenter de résumer les grosses caractéristiques de ces paramétrisations. On s'intéresse en général aux échanges verticaux, en négligeant les transferts horizontaux. Les échanges turbulents (flux de chaleurs sensible, latent et de quantité de mouvement) sont formulés à l'aide de la théorie de Monin-Obukhov appliquée entre la surface et un niveau de référence atmosphérique pris dans la couche limite de surface. Ce niveau de référence correspond généralement au niveau des mesures météorologiques $(2 \mathrm{~m}$ pour la température et l'humidité, $10 \mathrm{~m}$ pour la vitesse du vent). En modélisation atmosphérique, le niveau de référence est constitué par le premier niveau de calcul du modèle, niveau inférieur à 30 mètres. Les flux sont inversement proportionnels à la résistance aérodynamique. Celle-ci diminue lorsque la surface est rugueuse et que le gradient thermique dans la couche limite de surface est instable (gradient thermique inférieur à $\left.1^{\circ} \mathrm{C} / 100 \mathrm{~m}\right)$. Les échanges par rayonnement sont essentiels car ils déterminent l'énergie disponible. Les modèles les plus détaillés résolvent le transfert radiatif dans la végétation 
jusqu'à la surface du sol et permettent de déterminer la fraction du rayonnement incident solaire (de courtes longueurs d'onde) et atmosphérique (de grandes longueurs d'onde) absorbée par la végétation et la fraction atteignant le sol. Ce type d'approche entraîne le calcul de deux bilans radiatifs (pour le sol et pour la végétation), ce qui est particulièrement intéressant pour la représentation des couverts discontinus tels que les cultures en rang. Bien qu'il existe des modélisations extrêmement détaillées de la végétation, l'approche la plus fréquente en mode opérationnel revient à traiter la végétation comme une couche unique recouvrant partiellement le sol (concept 'big leaf' introduit par Deardorff en 1978) [24]. L'interception des précipitations ou de la rosée est modélisée à l'aide d'un réservoir de faible dimension (de l'ordre du $\mathrm{mm}$ pour une forêt tropicale). L'eau retenue par la végétation est ré-évaporée au taux potentiel (sans contrainte de la surface), sans atteindre la surface du sol. Lorsque la capacité maximale du réservoir est atteinte, l'eau ruisselle jusqu'au sol et s'ajoute à la partie des précipitations qui atteignent le sol directement. Le mécanisme de ré-évaporation de l'eau interceptée est particulièrement important dans le cas des couverts forestiers en raison de leur forte capacité d'interception mais aussi à cause de la valeur élevée de la longueur de rugosité (de l'ordre d'un mètre). Ainsi, on estime que 15 pour cent de l'évapotranspiration de la forêt tropicale est constituée par l'évaporation de l'eau interceptée. Ce mécanisme maintient un certain niveau d'humidité dans les basses couches de l'atmosphère pouvant entraîner un renforcement des précipitations [3] dans certains cas.

La modélisation de la transpiration de la végétation est un aspect très important en raison de son impact sur l'évolution des réserves en eau dans la zone racinaire. Généralement, la modélisation fait appel à la notion de résistance de surface s'ajoutant à la résistance aérodynamique et représentant les contraintes exercées par les plantes pour le transport de l'eau depuis les racines jusqu'aux cavités stomatales, siège de la transpiration. Plusieurs facteurs de contrôle sont pris en compte : le rayonnement solaire déclenchant la photosynthèse, la température de l'air dont les valeurs extrêmes limitent la transpiration, l'humidité de l'air, et surtout le contenu en eau du sol. Ce dernier facteur est déterminant pour le cycle annuel. En raison de la grande diversité du fonctionnement des plantes, il n'y a pas pour l'instant de consensus dans la représentation du stress hydrique. Les schémas les plus compliqués font appel à un calcul du potentiel foliaire (pression de l'eau dans les tissus végétaux) ainsi qu'à une distribution des racines permettant d'extraire l'eau dans les horizons du sol les plus humides [4]. Les méthodes plus simples utilisent des formulations empiriques pour la variation du stress entre deux valeurs caractéristiques du stock d'eau dans la zone racinaire : le point de flétrissement en deçà duquel l'eau n'est plus accessible par les plantes et la capacité au champ au delà de laquelle le sol n'exerce aucune contrainte sur le fonctionnement de la végétation. Ces formulations doivent être calibrées à l'aide de données expérimentales dont 1'obtention est peu aisée. La formulation de l'évaporation à la surface du sol fait elle aussi l'objet de nombreuses approches qui diffèrent lorsque le contenu en eau près de la surface devient faible [5]. L'évaporation est alors contrôlée par des mécanismes de diffusion de l'eau dans une fine pellicule de sol en surface, diffusion sous forme de vapeur. Les paramètres de contrôle sont associés à la granulométrie du sol et à ses propriétés hydrauliques près de la surface. Dans le cas d'un sol nu, une partie importante des apports de chaleur par rayonnement est transférée au sol par conduction thermique. Ainsi, le flux de chaleur conductif peut représenter $30 \%$ du rayonnement net pour un sol humide dont la conductivité thermique est élevée. La rugosité thermique du sol définit un niveau de référence pour la température de surface. Cette longueur de rugosité est très importante pour la répartition entre les flux de chaleur par convection vers l'atmosphère et par conduction vers le sol. En effet, lorsque la rugosité thermique est élevée, les échanges turbulents vers l'atmosphère sont privilégiés par rapport aux échanges thermiques [6].

Le traitement des échanges de chaleur et d'eau dans le sol peut être réalisé à partir des équations de diffusions (lois de Fourrier pour la température et de Darcy pour le contenu en eau) reliant l'évolution des quantités à la divergence de leurs flux verticaux. Les flux d'eau et de chaleur sont reliés aux gradients par l'intermédiaire de cœfficients de conductivité qui dépendent de la texture du sol et surtout du contenu en eau du sol. La conductivité hydraulique d'un sol varie de plusieurs ordres de grandeur lorsqu'on passe d'un sol sec à un sol humide. Ces très fortes non-linéarités compliquent le traitement numérique et nécessitent une forte résolution spatiale dans le sol, plus particulièrement près des conditions aux limites supérieure et inférieure.

Une autre technique simplifiée du traitement des échanges dans le sol est la méthode de rappel proposée par Deardorff [7]. Dans ce cas là, on considère deux réservoirs dans le sol. Le réservoir profond englobe le système racinaire et correspond au stock d'eau disponible dans le sol. Son évolution est estimée à partir des flux de surface (précipitations, évaporation), de l'extraction d'eau suite à la transpiration de la végétation et des écoulements de l'eau vers le système hydrologique (drainage et ruissellement). Le réservoir de surface permet de représenter les échanges d'eau et d'énergie pour une couche superficielle de quelques $\mathrm{mm}$. La modélisation des échanges entre les deux réservoirs fait appel à des cœefficients de calibration permettant de représenter les transferts par capillarité et par gravité [8].

La profondeur du sol considérée par le modèle est un paramètre important quelle que soit la complexité du schéma car elle détermine le volume total d'eau disponible pour les plantes.

La répartition du rapport évapotranspiration/ruissellement annuel est assez sensible à la valeur de la profondeur du sol.

Enfin, comme beaucoup de schémas sont conçus pour représenter les échanges sur de grandes mailles, quelques éléments de variabilité sous-maille sont représentés. La prise en compte d'une répartition statistique des précipitations convectives permet d'améliorer le traitement de l'interception dans la zone tropicale. Le ruissellement peut faire lui aussi l'objet d'un traitement particulier. Par exemple, la paramétrisation proposée par Dümenil et Todini [9] permettant de simuler une quantité d'eau ruisselée avant que toute la maille soit saturée (et ceci d'autant plus que la distribution du relief dans la maille est forte) a conduit à une amélioration nette du bilan hydrique de surface dans certains modèles du climat.

La prise en compte de la variabilité de la végétation et du sol peut être schématisée par deux approches : soit le calcul des échanges est réalisé pour l'ensemble des divers types de surface qui constituent la grande maille et les flux moyens sont estimés à partir de la moyenne des flux élémentaires ; soit on estime des paramètres effectifs et un seul flux effectif pour toute la maille. 


\section{- 2.2 Validation et intercomparaison des schémas de surface}

La validation des schémas de surface est une procédure complexe en raison de la disparité des processus physiques à examiner mais aussi parce que la mise en forme de données expérimentales suffisamment complètes n'est pas facile. Le plus simple est d'examiner la capacité du modèle à reproduire les échanges de surface à l'échelle locale, c'est-à-dire à l'échelle de la parcelle, considérée comme homogène horizontalement et pour laquelle on sait mesurer les flux de surface par diverses méthodes $|10|$. Il faut ensuite connaitre les propriétés du sol (profondeur du sol, texture du sol, propriétés thermohydriques si on utilise un modèle détaillé) et de la végétation (longueur de rugosité, albédo, fraction de recouvrement, indice foliaire, paramètres de la résistance de surface dépendant du type de végétation,....). Les réservoirs du sol sont initialisés à l'aide d'observations de température et de contenu en eau du sol. Ce dernier point est très important si on veut espérer réaliser une bonne calibration de la relation eau du sol/évaporation. Le modèle est ensuite forcé par les quantités micrométéorologiques observées (température, humidité, vitesse du vent, précipitations, rayonnements solaire et atmosphérique) et simule les flux de surface ainsi que l'évolution de la température et de l'eau du sol. Ces quantités modélisées sont comparées aux observations des flux et de l'eau obtenues simultanément sur le même site. On peut ainsi calibrer un schéma sur un type de sol et de végétation donné en procédant par minimisation des erreurs de simulation des flux de surface. La figure 1 propose une illustration de calibration du schéma ISBA utilisé à Météo-France pour deux types de surface contrastés : un cas de sol nu en Espagne et un site de forêt en Amazonie. Deux belles journées sont choisies caractérisées par de fortes valeurs d'apports radiatifs (rayonnement net d'environ $700 \mathrm{~W} / \mathrm{m}^{2}$ à midi). Dans le cas du sol nu et sec, les flux de chaleur sensible et conductif sont prépondérants et l'évaporation de surface reste faible sur la journée. En revanche, le

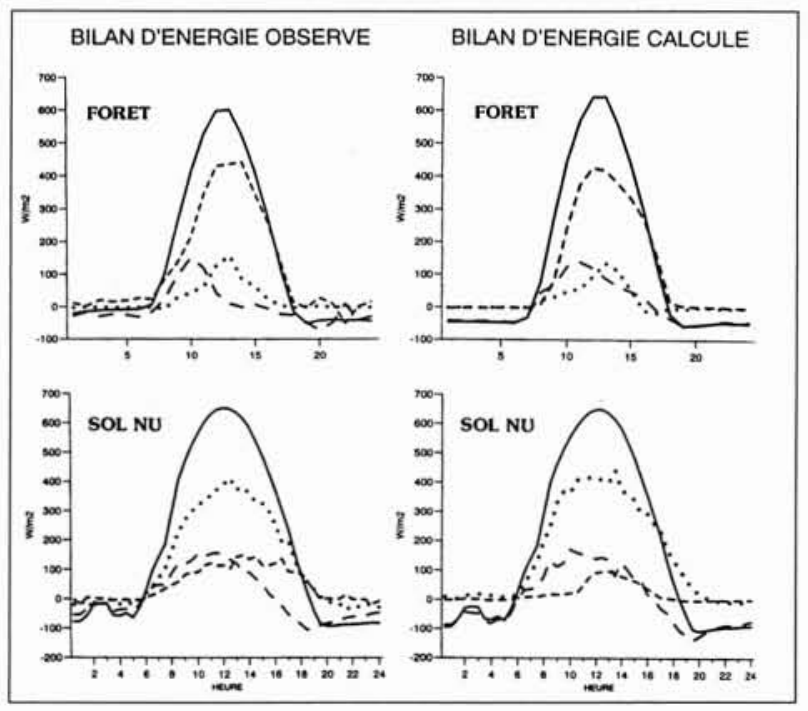

1. Observations et simulations des variations diurnes des flux de surface sur un site de forêt tropicale (Expérience ABRACOS en Amazonie) et sur un site de sol nu (Expérience EFEDA en Espagne) : flux de rayonnement net (trait continu), flux de chaleur latente (tiretés courts), flux de chaleur sensible (points), flux par conduction (tiretés longs). Les simulations sont réalisées par le schéma ISBA (Interactions entre le Sol, la Biosphère et l'Atmosphère) site forestier fait apparaître des valeurs très élevées du flux de chaleur latente correspondant à la transpiration des plantes.

L'effort consenti ces dix dernières années lors de grandes campagnes de mesures internationales a permis de collecter des données de surface extrêmement diversifiées recouvrant la plupart des formations végétales présentes sur la surface du globe : forêt tropicale, savanes et zones déforestées en Amazonie (programmes ARME, 1984-85 et Abracos, 1990-93, [11]), prairie, cultures et forêts aux latitudes tempérées (programmes FIFE, 1987, et Hapex-Mobilhy 1985-86, [23], Heife 1991), végétation des zones semi-arides (programmes EFEDA, 1991, |12| et Hapex-Sahel 1992, |13|) et enfin, végétation boréale aux hautes latitudes (programme BOREAS 1994-95, [27|). On dispose ainsi d'une bonne caractérisation expérimentale des échanges d'eau et de chaleur à l'interface sol-plante-atmosphère pour des périodes de quelques mois.

En revanche, peu de données sont disponibles sur le long terme. Or, certains aspects du bilan hydrique ne sont accessibles que sur la base de l'année. A cette échelle, on peut considérer que le stockage ou bien le déstockage de l'eau dans le sol est faible. Les apports par précipitation vont être équilibrés par les pertes d'eau par évapotranspiration, drainage et ruissellement. L'équilibre de ces flux d'eau est contrôlé par les caractéristiques du sol et de la végétation (profondeur du sol, cycle de la végétation, ...). Un des objectifs du programme d'intercomparaison des schémas de surface, le programme PILPS [14], est d'analyser les facteurs qui conditionnent le bilan hydrique annuel. Les premiers résultats de PILPS montrent que la réponse d'une vaste gamme de schémas est assez dispersée, même lorsqu'on utilise un forçage atmosphérique et des paramètres de surface identiques. Cette dispersion est due à l'absence de formulation unifiée des processus hydriques. Par exemple, il y a encore beaucoup d'empirisme dans la modélisation de la transpiration de la végétation, en particulier en présence d'un stress hydrique marqué. Une façon de progresser dans les formulations de tous ces processus complexes est de réunir des jeux de données sur une longue durée avec une documentation aussi complète que possible des échanges atmosphériques, hydrologiques et biologiques. Quelques jeux de données sont déjà disponibles tel que celui obtenu sur un site de prairie aux Pays Bas, ayant servi de référence dans une étape du programme d'intercomparaison PILPS [15].

\section{III $\square$ LA MODÉLISATION DE L'EAU À MOYENNE ÉCHELLE}

\section{- 3.1 La modélisation atmosphérique à mésoéchelle}

La compréhension du cycle de l'eau à l'échelle régionale a constitué l'objectif des grandes campagnes expérimentales conduites au cours de la dernière décennie avec deux objectifs majeurs :

1) permettre une estimation du bilan hydrique à l'échelle régionale afin de tester les schémas de surface intégrés dans les modèles de circulation climatique.

2) comprendre les processus physiques de circulation de l'eau à moyenne échelle en relation avec la variabilité des états de surface.

De telles expériences considèrent de grands domaines $(100 \mathrm{~km}$ par $100 \mathrm{~km})$ comparables aux dimensions d'une maille de modèle climatique. Un dispositif expérimental complexe est mis en œuvre pour estimer les flux régionaux avec 
une attention particulière au cycle diurne. Les flux de surface sont mesurés sur un ensemble de types de surface représentatifs de la zone étudiée : sites de cultures et forêt Landaise dans le cadre d'Hapex-Mobilhy, sol nu, vignes et cultures irriguées en Espagne pendant EFEDA, savane, jachère arborée et brousse tigrée au Niger dans le cadre d'Hapex-Sahel, pâturage et forêt tropicale au Brésil dans le cadre d'Abracos. Chaque site de surface est équipé d'un dispositif très complet permettant d'accéder aux divers facteurs des bilans radiatif, énergétique et hydrique de la surface, à l'eau dans le sol et au fonctionnement de la végétation (mesures de l'indice foliaire, de la résistance stomatique, de la hauteur, ...). Un réseau complémentaire de mesures atmosphériques est déployé : radiosondages dans la couche limite atmosphérique en deux ou trois points du domaine et mesures aéroportées des flux dans l'atmosphère suivant un plan de vol prédéterminé par les inhomogénéités de la surface. Enfin, la télédétection complète cet arsenal instrumental en apportant une vision à diverses échelles des signatures radiatives permettant d'identifier les zones de végétation (par exemple indice de végétation de type NDVI exploitant les réflectivités du sol et de la végétation dans le spectre solaire) et d'estimer certains paramètres de contrôle de l'évaporation (indice foliaire, albédo de la surface,....). Dans une telle campagne expérimentale, la modélisation atmosphérique à méso-échelle représente un outil nécessaire pour l'interprétation et l'agrégation spatiale des données collectées. Partant de conditions aux limites bien définies (analyses opérationnelles enrichies de radiosondages pris dans la zone de la mesure, carte du sol et de la végétation, contenu en eau du sol,...), le modèle de mésoéchelle est intégré afin de simuler le cycle diurne de quelques journées sélectionnées. Cette méthodologie a été bien suivie dans le cadre d'Hapex-Mobilhy, d'EFEDA et d'Hapex-Sahel. On a pu ainsi mettre en évidence certains processus de recyclage de l'eau au sein du domaine. Par exemple, l'effet de l'interception des précipitations par la forêt Landaise sur le renforcement des précipitations stratiformes a été étudié par la modélisation couplée de l'atmosphère et des processus de surface [3]. Dans ce cas, le modèle a été utilisé pour préciser l'importance de la rugosité de la surface et la capacité d'interception sur le renforcement des précipitations.

Les mécanismes de brises thermiques déclenchées par des contrastes de bilan d'énergie à la surface constituent une autre gamme de processus qui ont pu être observés et simulés dans le cadre de ces diverses campagnes de mesures. Ainsi au début de l'été, le contraste d'échauffement entre la forêt des Landes et les cultures avoisinantes peut déclencher une circulation thermique dirigée des cultures vers la forêt. Cette circulation transporte de l'air humide vers la forêt pouvant entraîner la formation de nuages de couche limite dans certaines conditions. Grâce à une bonne description des processus de surface dans le modèle de mésoéchelle, il a été possible de simuler avec un certain succès les cumulus de beau temps fréquemment observés sur la forêt Landaise [16]. D'autres études de modélisation plus académiques ont quantifié les circulations de brises produites par des contrastes de rugosité de surface (transition forêt/cultures) ou de contenu en eau du sol [29].

L'expérience Hapex-Sahel a permis l'étude d'un autre aspect de la circulation atmosphérique de l'eau à des échelles spatiales relativement faibles. En effet, il existe un fort couplage entre les précipitations et l'évapotranspiration liés à l'importance de la convection sur le cycle de l'eau à ces latitudes. L'interdépendance des cultures et du cycle de l'eau atmosphérique, incluant les précipitations et leur variabilité spatiale sont au cœur des recherches menées sur le Sahel. La figure 2 illustre la variabilité spatiale du flux d'évaporation mesuré à 50 mètres du sol par un avion instrumenté dans le nord de la zone expérimentale Hapex-Sahel [17]. Cette carte fait apparaître une variation du flux de plus de $150 \mathrm{~W} / \mathrm{m}^{2}$ entre le nord et le sud, différence qui peut être reliée au gradient important des précipitations cumulées sur la zone. II faut également souligner l'occurrence d'un orage important dans le sud du domaine la journée précédant la mesure aéroportée. Cet apport de précipitations assez localisé explique vraisemblablement le maximum d'évaporation observé dans le sud est du domaine (figure 2).

Mais le cycle de l'eau régional est lui-même modifié par la circulation à plus grande échelle. Dans le cadre d'EFEDA, l'effet de la brise de mer sur le transport vertical de l'humidité sur les plateaux de Castille a pu être montré à partir de la modélisation. Ainsi le transport vertical de l'eau dans l'atmosphère n'est pas strictement lié aux processus d'évaporation de la surface mais résulte de l'interaction avec une circulation organisée à l'échelle synoptique.

Après avoir été validés sur quelques situations, les modèles de mésoéchelles peuvent être utilisés comme des laboratoires numériques pour explorer certains aspects de la variabilité spatiale de la surface, ce qui représente une question posée dans les modèles de simulations du climat. Noilhan et Lacarrère $[18]$ ont montré qu'il est raisonnable de prendre en compte cette variabilité à partir de paramètres effectifs de la végétation et du sol, sous réserve de moyenner les propriétés de surface (longueur de rugosité, fraction de végétation, albédo, indice foliaire, résistance de surface) avec des opérateurs qui conservent les flux de surface de quantité de mouvement (moyenne du logarithme des rugosités), de l'évaporation (moyenne arithmétique des indices foliaires et

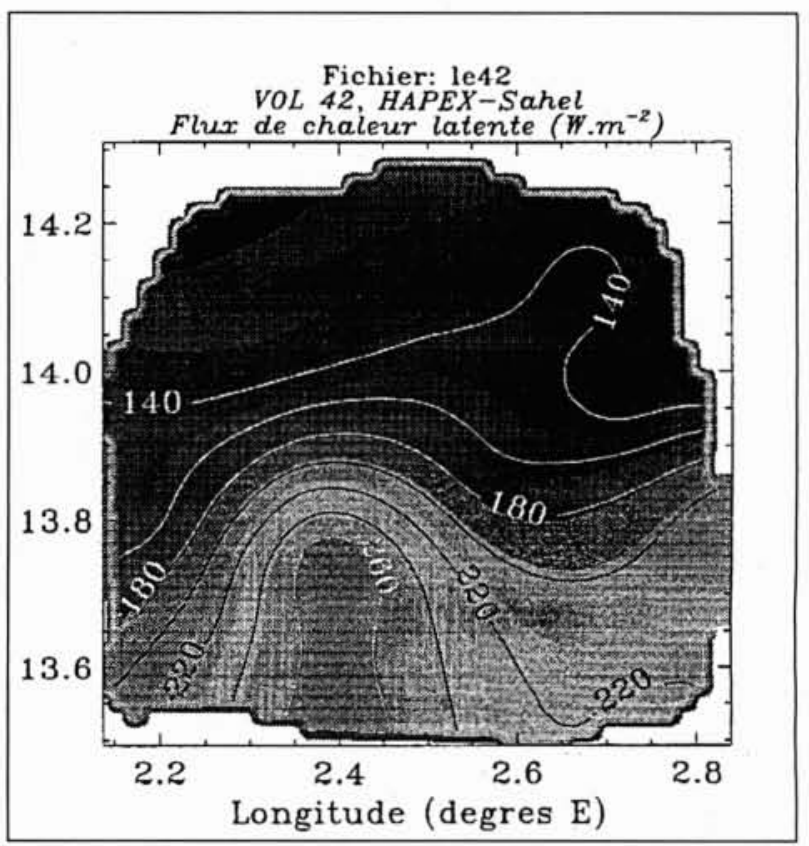

2. Variations du flux de chaleur latente mesuré par un avion instrumenté (le Merlin IV de Météo-France) au Niger dans le cadre de l'expérience Hapex-Sahel. Le champ de l'évaporation à été obtenu à partir des mesures aéroportées réalisées à $\mathbf{5 0}$ mètres au-dessus de la surface à la mi-journée du 12 septembre 1992 [17]. 
moyenne des inverses des résistances de surface) et des flux radiatifs (moyenne des albédos de surface). La méthode a été testée avec succès sur quelques jours sur la zone HapexMobilhy constituée de la forêt Landaise et de cultures en conditions humides, mais aussi en conditions plus sèches en Espagne dans le cadre d'EFEDA. Ainsi, il semble que l'on puisse représenter la variabilité de la végétation de façon relativement simple et que cette approche soit préférable à l'hypothèse souvent utilisée de considérer les propriétés associées au type dominant. Cependant, le traitement du sol par cette méthode peut introduire des erreurs importantes en raison des très fortes non-linéarités existant entre le flux d'évaporation et le contenu en eau du sol. Un premier élément de prise en compte de la variabilité des sols sur le flux d'évaporation est l'approche stochastique qui reste cependant limitée à des échelles spatiales relativement faibles (échelle de l'unité pédologique).

\section{- 3.2. Vers un couplage de la modélisation atmosphé- rique et hydrologique à l'échelle régionale}

Si l'on veut décrire complètement la partie continentale du cycle de l'eau, il faut développer une modélisation couplée qui puisse traiter les échelles de temps et d'espace caractéristiques du transport de l'eau dans l'atmosphère et les systèmes hydrologiques. A moyenne échelle, les quantités atmosphériques possèdent une grande variabilité temporelle liée au cycle diurne et une variation horizontale assez lisse. La résolution spatiale d'un modèle atmosphérique de mésoéchelle est de quelques $\mathrm{km}$ et son pas d'intégration temporelle de quelques minutes. En revanche, le milieu hydrologique se caractérise par une grande variabilité spatiale des caractéristiques du milieu (variations géologiques, pédologiques, topographiques,...) et une variabilité temporelle assez faible à

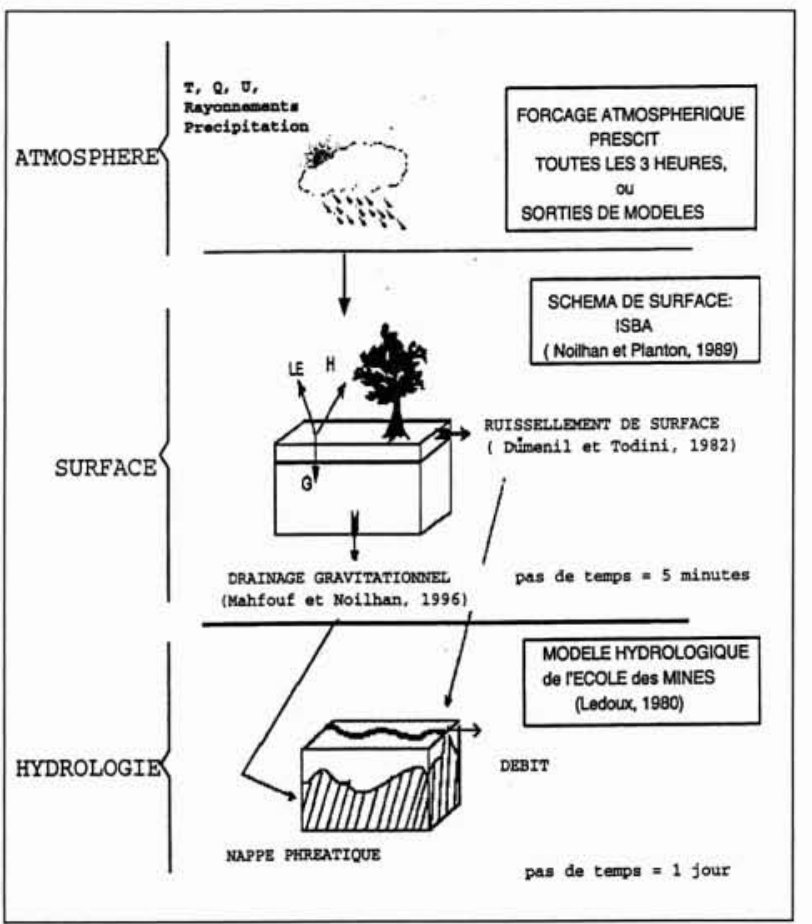

3. Schématisation des éléments de couplage entre un modèle atmosphérique et un modèle hydrologique distribué à l'échelle régionale [21]. Ce projet de couplage est développé dans le cadre du programme GEWEX-Rhône. l'échelle diurne et marquée par le cycle hydrologique annuel. En raison de ce contraste des échelles de temps, il existe peu d'exemples à notre connaissance de couplage entre modèles atmosphérique et hydrologique à moyenne échelle. Or les perspectives de changements climatiques posent le problème de l'impact des modifications du régime des précipitations et de la distribution de la température de l'air sur la ressource en eau régionale. Cela souligne tout l'intérêt de mettre en place une modélisation couplée à mésoéchelle. Tel est l'objectif du programme GEWEXRhône qui vise à développer un système de modélisation couplée sur un grand bassin versant possédant une composante nivale potentiellement sensible à un réchauffement du climat [19]. La première étape du couplage concerne le développement d'une interface commune aux modèles hydrologiques (le modèle hydrologique distribué du CIG, [20]) et atmosphérique. Le schéma ISBA assure cette interface (figure 3). La partie aérienne du schéma de surface permet le couplage avec l'atmosphère à travers la résolution explicite du cycle diurne des bilans d'énergie et hydriques avec un pas de temps de quelques minutes. Il s'agit là d'une modification importante de la version initiale du modèle d'hydrologie qui possédait des fonctions de production fonctionnant au pas de temps journalier. La partie souterraine du schéma ISBA assure l'alimentation en eau du système hydrologique : drainage gravitationnel vers les aquifères souterrains, ruissellement vers le réseau de drainage. Les flux de drainage et de ruissellement sont cumulés sur la journée et transférés au réseau de drainage maillé du modèle hydrologique. Le système couplé peut être intégré sur le long terme (e.g. le cycle annuel) si I'on sait intégrer un modèle atmosphérique de mésoéchelle sur une longue durée avec des conditions aux limites fournies par un modèle coupleur de grandes échelles. Une autre approche consiste à utiliser des observations ou des prévisions pour fournir les paramètres de forçages atmosphériques au niveau de référence (paramètres moyens, flux radiatifs, précipitations). Dans ce cas, on ne tient pas compte de la rétroaction des processus de surface sur l'évolution des quantités atmosphériques. Une telle approche est actuellement à l'étude sur le bassin de l'Adour, siège de l'expérience Hapex-Mobilhy [21]. Dans cette analyse, le potentiel de la modélisation est évalué à travers la simulation du cycle diurne et saisonnier de l'évaporation de la surface (ce qui nécessite une bonne description de la végétation et des sols dans le domaine) et à travers la simulation des débits journaliers. Les autres aspects importants de ce programme sont le développement d'un schéma décrivant la neige et les glaciers ainsi que la prise en compte de toutes les classifications à haute résolution spatiale sur la végétation (base de données Corine Land Cover) et les sols en France (carte des sols de l'INR, [22]).

\section{IV $\square$ CONCLUSIONS}

Ce panorama assez général a mis en lumière la complexité des mécanismes physiques à modéliser pour une meilleure maîtrise de notre connaissance des ressources en eau régionales. A l'échelle locale, on dispose d'une vaste palette de modélisation des échanges à l'interface sol-plante-atmosphère ainsi que de très nombreux jeux de données permettant d'évaluer les performances des modèles sur le cycle diurne des flux de surface. Lorsqu'on considère le cycle annuel, on note une assez grande dispersion des simulations qui semble liée aux imperfections de la modélisation du cycle de l'eau 
dans le sol. La constitution de jeu de données sur le long terme est une piste pour progresser dans la modélisation.

La modélisation atmosphérique à mésoéchelle permet de comprendre les processus de circulation de l'eau en relation avec les échanges de surface : influence de l'interception sur les précipitations, couplage entre les précipitations et l'évaporation à la surface, circulation de mésoéchelle provoquée par des contrastes de surface. A ce jour, la branche hydrologique du cycle de l'eau est modélisée sans couplage réel avec la branche atmosphérique, le forçage par les précipitations et l'évaporation potentielle étant imposé comme conditions aux limites. Un effort est actuellement consenti dans notre communauté pour développer un système de modélisation couplé à mésoéchelle appliqué à un grand bassin fluvial. Les grands traits de ce programme sont résumés en fin d'article.

\section{Bibliographie}

[1] Royer J.F. , 1989: Circulation atmosphérique et sécheresse; La Houille Blanche, 7/8, 497-504.

[2] Choisnel E., 1992 : Le calcul du bilan hydrique du sol: options de modélisation et niveaux de complexité. Sciences du sol, 30, 15-31.

[3] Blyth E., Noilhan J et Dolman A.J., 1993 : Sensitivity of mesoscale circulations to surface wetness: an example from Hapex-Mobilhy. J. Appl. Meteorol., 33, 445-454.

[4] Braud I., Angulo-Jaramillo, M. Vauclin, J.L. Thony et Ruelle P., 1995 : A simple soil-plant-atmosphere transfer model (Sispat) . Development and field verification. J. Hydrol. , 166, 213-250.

[5] Mahfouf J.F. et Noilhan J., 1991 : Comparative study of various formulations of evaporation from bare soil using in-situ data. J. Appl. Meteor., 30, 1354-1365.

[6] Giordani, H., Noilhan J., Lacarrère P., Bessemoulin P. et Mascart P., 1996 : Modelling the surface processes and the atmospheric boundary layer for semiarid conditions. Agri. Forest Meteorol. (in press)

[7] Deardorff, J. W. 1977 : A parameterization of groundsurface moisture content for use in atmosphere predictions models. J. Appl. Meteor., 16, 1182-1185.

[8] Noilhan, J. and Planton, S. 1989 : A simple parameterization of land surface processes for meteorological models. Mon. Wea. Rev., 117, 536-549.

[9] Dümenil L. et Todini E., 1992 : A rainfall-runoff scheme for use in the Hamburg Climate Model. in J.P. O'Kane editor:'Advances in theoretical hydrology- A tribute to James Dooge', Elsevier, Amsterdam.

[10] Bessemoulin P., 1993 : Méthodes expérimentales de détermination des flux de surface. Météo-France, note numéro 6 du Groupe de Météorologie expérimentale et instrumentale, Toulouse.

[11] Gash J., Nobre C.A., Roberts J.M. et Victoria R.L., 1996 : An overview of ABRACOS. In Amazonian Deforestation and Climate, édité par J.H.C. Gash, C.A. Nobre, J.M. Roberts et R.L. Victoria, Wiley, p. 1-14.

[12] Bolle et 36 co-auteurs, 1993 : EFEDA: European Field Experiment in a Desertification Threatened Area. Annales Geophysicae, 11, 173-189.

[13] Goutorbe et 15 co-auteurs, 1994 : HAPEX-Sahel: A large-scale study of land-atmosphere interactions in the semi-arid tropics. Annales Geophysicae, 12, 53-64.

[14] Henderson-Sellers, A., Pitman,A.J., Love, P.K.,
Irannejad, P. and Chen, T. 1995 : The project for intercomparison of land surface parameterization schemes (PILPS): Phases 2 and 3. Bull. Amer. Meteorol. Soc., 76, 489-503.

[15] Chen T.H. et 31 co-auteurs, 1996 : Cabauw experimental results from the project for intercomparison of landsurface parameterization schemes (PILPS) . Journal of Climate, (In press)

[16] Bougeault Ph., Bret B., Lacarrère P. et Noilhan J., 1991 : An experiment with an advanced surface parameterization in a meso-beta-model. part II: The 16 June 1986 simulation. Mon. Wea. Rev., 119, 2374-2392.

[17] Saïd F., Attié J.L., Bénech B., Druilhet A., Durand P., Marciniak M. H. et Monteny B., 1996 : Spatial variability in airborne surface flux measurements during HapexSahel. J. Hydology, Hapex-Sahel special issue (in press)

[18] Noilhan J. et Lacarrère P., 1995 : GCM gridscale evaporation from mesoscale modelling. J. of Climate, 8, 206-223.

[19] Vidal-Madjar D., et 10 co-auteurs, 1995 : Modélisation couplée d'un grand bassin (Rhône). Programme Gewex/hydrologie continetale du programme du PNEDC (Disponible sur demande auprès de J. Noilhan, CNRM, Toulouse 31057).

[20] Ledoux E., 1980 : Modélisation intégrée des écoulements de surface et des écoulements souterrains sur un bassin hydrologique. Thèse de Docteur-Ingénieur, Université Pierre et Marie Curie, Paris VI (19 décembre 1980).

[21] Habets F., Lacarrère P., Noilhan J., Péris P., Ledoux E., Vidal-Madjar D. et Ottlé C., 1995 : Eléments de couplage d'un modèle atmosphérique à mésoéchelle avec un modèle hydrologique: Application sur le bassin de l'Adour dans le cadre de l'expérience HapexMobilhy 86. Atelier de modélisation de l'atmosphère, Météo-France, CNRM, Décembre 1995, 269-278.

[22] King D., Jones R.J.A. et Thomasson A.J., 1995 : European land information systems for Agro-Environmental Monitoring. European Commission, DG XIII.

[23] André et 30 co-auteurs, 1988 : Evaporation over land surfaces: First results from Hapex-Mobilhy special observing period, Annales Geophysicae, 6, 477-492.

[24] Deardorff J.W., 1978 : Efficient prediction of ground surface temperature and moisture with inclusion of a layer of vegetation. J. Geophys. Res., 20, 1889-1903

[25] Ducoudré N., Laval K. et Perrier A., 1993 : SECHIBA, a new set of parameterizations of the hydrologic exchanges at the land / Atmosphere interface within the LMD atmospheric general circulation model. J. Climate, 6, 248-273.

[26] Sellers P et 4 co-auteurs, 1992 : An overview of the First International Satellite Land Surface Climatology Project (ISLSCP) Field Experiment (FIFE). J. Geophys. Res., 97, 18345-18373.

[27] Sellers P.,et 5 co-auteurs, 1995 : The Boreal Ecosystem-Atmosphere Study (BOREAS) : An overview and early results from the 1994 field year, Bull. Amer. Meteorol. Soc., 76, 1549-1577.

[28] Choisnel E., 1996 : Le cycle de I'eau. Ed. INRA, coll. "Le point sur" (à paraître).

[29] Mahfouf J. F. , Richard E. et Mascart P. , 1987 : The influence of soil and vegetation on the development of mesoscale circulations. 